\title{
Ba Duan Jin and the Treatment of Illness in General, and Cognitive Impairment in Particular
}

\author{
Robert W McGee* \\ Department of Graduate and Professional Studies in Business, Fayetteville State University, USA \\ *Corresponding author: Robert W McGee, Department of Graduate and Professional Studies in Business, Fayetteville \\ State University, USA
}

\section{ARTICLE INFO}

Received: November 21, 2021

Published: November 29, 2021

Citation: Robert W McGee. Ba Duan Jin and the Treatment of Illness in General, and Cognitive Impairment in Particular. Biomed J Sci \& Tech Res 40(2)-2021. BJSTR. MS.ID.006424.

\section{ABSTRACT}

This article cites numerous studies that have found qigong and tai chi to be effective in treating a wide range of illnesses, then proceeds to review several studies where $\mathrm{Ba}$ Duan Jin, a popular set of qigong exercises and a tool of traditional Chinese medicine (TCM), has been used to treat cognitive impairment.

Keywords: Qigong; Tai Chi; Taiji; Ba Duan Jin; Baduanjin; TCM; Traditional Chinese Medicine; Treatment of Disease; Cognitive Impairment

Abbreviations: TCM: Traditional Chinese Medicine; RCTs: Randomized Control Trials; CI: Cognitive Impairment; MMSE: Mini-Mental State Examination; MoCA: Montreal Cognitive Assessment; CSR: Cervical Spondylotic Radiculopathy; PCI: Percutaneous Coronary Intervention; CS: Cervical Spondylosis

\section{Introduction}

Qigong (pronounced chee gong) is a tool in the toolbox of traditional Chinese medicine (TCM). It is used to treat and even cure a wide range of diseases [1-54]. Ba Duan Jin (baduanjin) is the most popular set of qigong exercises. Zhang, et al. [55] examined the results of 886 clinical studies in 14 countries and discovered that Ba Duan Jin was used in 492 (55.5\%) of them. Some of the diseases and ailments that have been treated with qigong exercises include:

i. Ankylosing Spondylitis [56]

ii. Anxiety [38,57-58]

iii. Arthritis [39,59-61]

iv. Attention Deficit [62]

v. Autism [63]

vi. Back Pain [64]

vii. Blood Pressure [40,65-66]

viii. Cancer $[36,47,67-84]$ ix. Chronic Fatigue Syndrome, Cognitive Impairment and COPD [85-101]

x. Covid-19 [46,102-104]

xi. Depression [38,105-114]

xii. Fibromyalgia [115]

xiii. Frailty [116-118]

xiv. Heart Disease [119]

xv. Hypertension [40,65-66]

xvi. Immune System [120]

xvii. Parkinson's Disease [121-126]

xviii. Quality of Life [127-131]

xix. Rheumatism [132]

xx. Schizophrenia [133]

xxi. Stress [134] 
xxii. Stroke [135-138]

xxiii. Substance Abuse [139]

xxiv. Unilateral Vocal Fold Paralysis [140]

Ba Duan Jin consists of a series of 8 qigong exercises. The version promoted by the International Health Qigong Federation [9] takes about 12 minutes to perform. Qigong is similar to yoga, in that it involves physical movement, breathing and moving meditation, but it is not yoga. Qigong is actually gentler than yoga. It is a close cousin of kung fu and other martial arts, but it is not quite a martial art. It involves unblocking the flow of energy in the body, like acupuncture and acupressure, but it is not acupuncture or acupressure. It is an internal component of tai chi, which is a martial art. When one does tai chi properly, one is also doing qigong, perhaps unknowingly. It is a form of meditation as well as exercise, and can be performed from a standing or seated position.

\section{Ba Duan Jin Studies}

\section{Wang, et al. (2021) [141]}

Wang, et al. [141] systematically evaluated the effects of Baduanjin on global cognitive function and specific cognitive domains of middle-aged and elderly individuals. They searched multiple data bases, looking for randomized control trials (RCTs) that utilized Baduanjin exercises. They found that Baduanjin exercises resulted in significant benefit for global cognitive function and parts of specific domains of cognition, including immediate and delayed memory, executive function, and processing speed. However, no significant difference was found in attention function, visual-spatial ability or long-term memory. None of the studies reported any adverse effects. They concluded that Baduanjin is safe and effective in enhancing global cognitive function and memory, and might be beneficial for other cognitive domains, such as executive function and processing speed.

Eleven of the RCTs compared Baduanjin to non-exercise control; 3 compared Baduanjin and other no-exercise treatments to the same no-exercise treatment. Duration of the studies varied between 1.5 and 12 months. Frequency of the sessions varied between 3-7 per week. Sessions lasted 30-60 minutes. In most studies, participants were older than 60 . In four studies, participants were between 45 55. Global cognitive function was tested in 13 studies that included 938 participants. Cognitive function was measured by the MMSE, MoCA and LOTCA scales. The results from 6 pooled studies of 444 participants found that Baduanjin significantly improved the MMSE scores without heterogeneity $(\mathrm{p}<0.001)$. In 9 other studies involving a total of 628 participants, it was found that Baduanjin improved MoCA scores, although heterogeneity was present among the studies ( $\mathrm{p}<0.001)$. A smaller study involving 60 participants measured global cognition using the LOTCA scale. That study found significant improvement $(\mathrm{p}<0.001)$.

Specific cognitive domain was also examined. Significant improvement was found in general memory function in two studies involving 157 participants ( $\mathrm{p}<0.001$ ). Four studies examining immediate memory showed significant improvement $(p<0.001)$ in the Baduanjin group compared to the control group. Several studies of delayed memory found that MD values increased significantly in the Baduanjin group. Two studies involving 109 participants found that the Baduanjin group's executive function using the TMT improved significantly ( $\mathrm{p}=0.05$ ) over that of the control group. However, Baduanjin had no significant effect on the Go/No Go reaction-time test and the correct-number test. Two studies on processing speed found that Baduanjin significantly improved DSC scores ( $p=0.0008$ ). One study on the effects of Baduanjin on visualspatial ability found no significant difference between the Badjanjin and control groups. No serious adverse events were reported by any of the studies during the Baduanjin training. The findings suggest that Baduanjin is safe and effective for enhancing global cognitive function and memory in middle-aged and older adults, and may benefit other cognitive functions.

\section{Yu, et al. (2020) [142]}

Yu, et al. [142] reviewed 16 randomized control trials (RCTs) involving 1054 participants on the effect of Baduanjin on patients having mild cognitive impairment. They found that Baduanjin combined with conventional therapy produced significantly better results than conventional therapy alone after six months of treatment in terms of the Montreal Cognitive Assessment and Mini-Mental State Examination scores ( $p<0.00001)$. There was also significant improvement in some dimensional scores on the Wechsler Memory Scale and the auditory verbal learning test scores after six months $(\mathrm{p}<0.05)$.

\section{Zheng, et al. (2020) [143]}

Zheng, et al. [143] conducted a randomized control trial (RCT) on the effects of Baduanjin on cognitive function in patients with post-stroke cognitive impairment. It was a randomized, two-arm parallel controlled trial with allocation concealment and assessors blinding, and was conducted in the community center of Fuzhou city, China. Forty-one participants completed the study (22 Baduanjin and 19 control group), which consisted of 24 weeks of Baduanjin training, 3 days a week, 40 minutes per day. The control group maintained their original medication and rehabilitation regimen. Mean scores were significantly different between the two groups for global cognitive function, execution, memory (immediate recall), short-term and long-term delayed recognition, attention response time, and activities of daily living. The study concluded that regular Baduanjin training is associated with less loss of cognitive function in patients after a stroke. 


\section{Li, et al. (2021) [144]}

Li et al. [144] studied the effects of four kinds of traditional Chinese exercise (TCE) on patients with cognitive impairment. They found that Baduanjin may be the most effective of the four exercises for significantly improving cognitive function, followed by tai chi, Liuzijue and qigong. They examined 27 randomized control trials (RCTs) involving 2414 patients with sample sizes ranging from 10-194. The groups consisted of 1133 in the TCE groups and 1281 in the control groups. The breakdown of the 4 TCE groups was as follows:

i. Tai chi 644

ii. Baduanjin 386

iii. Liuzijue 75

iv. Qigong 28

Participants had the following diagnoses:

i. Dementia 4

ii. Mild cognitive impairment 17

iii. Cognitive impairment $(\mathrm{CI}) 6$

The RCTs were conducted in the following countries:

i. China 22

ii. Thailand 1 iii. USA 1

iv. England 1

v. France 1

vi. Not disclosed 1

The intervention lengths varied from 7 weeks to 25 months, between 1 and 6 times per week, from 30 to 90 minutes per day. The scales used for cognitive assessment were the Mini-Mental State examination (MMSE), the Chinese version (CMMSE), and the Montreal Cognitive Assessment (MoCA). Pairwise comparisons of the four types of TCE found that all four had significant improvements in global cognition, as measured by the MMSE or MoCA. The $p$-values for the four TCEs were:

i. Baduanjin $\mathrm{p}<0.00001$

ii. Tai Chi $\mathrm{p}<0.00001$

iii. Liuzijue $\mathrm{p}=0.003$

iv. Qigong $\mathrm{p}=0.02$

Li, et al. [144] ranked the probability of the efficacy of the different interventions. Baduanjin was most likely to rank first (53\%); tai chi was most likely to rank second (40\%), etc. The full rankings, taken from the study, are given below. Li, et al. [144] cited several other studies that reached similar conclusions regarding the use of Baduanjin and tai chi to improve cognitive function [145147] (Table 1).

Table 1: Rank Probability of the Efficacy of Different Interventions.

\begin{tabular}{|c|c|c|c|c|}
\hline Intervention & Rank 1 & Rank 2 & Rank 3 & Rank 4 \\
\hline Baduanjin & 0.53 & 0.32 & 0.12 & 0.02 \\
\hline Control & 0.00 & 0.00 & 0.06 & 0.38 \\
\hline Liuzijue & 0.14 & 0.15 & 0.26 & 0.27 \\
\hline Qigong & 0.22 & 0.13 & 0.20 & 0.20 \\
\hline Tai Chi & 0.12 & 0.40 & 0.35 & 0.13 \\
\hline
\end{tabular}

\section{Concluding Comments}

It is clear that Baduanjin and other traditional Chinese exercises can aid in the treatment of cognitive decline. Many studies have found that TCE can be beneficial in the treatment of many other ailments as well. Several studies are now in process that are examining the effects of Baduanjin on other ailments. The results of those studies are not yet available as of this writing. Chen, et al. [148] searched several data bases to find controlled trials that evaluated the effects of Baduanjin on postoperative rehabilitation of breast cancer patients. The goal of their study will be to offer a guideline for clinical workers. The results have not been published as of this writing. Dai, et al. [149] are searching several databases to determine the effectiveness of baduanjin on the treatment of cervical spondylotic radiculopathy (CSR). Li, et al. [150] plan to conduct a systematic review and meta-analysis to determine whether Baduanjin is an effective intervention in post percutaneous coronary intervention (PCI) patients. Zou, et al. [151] are conducting a study, the aim of which is to evaluate the safety and effectiveness of Baduanjin for patients having cervical spondylosis (CS).

\section{Conflict of Interest}

There is no conflict of interest.

\section{Funding}

This study was not funded. 


\section{References}

1. American Academy for Medical Qigong.

2. American Qigong Association.

3. American Tai Chi and Qigong Association.

4. British Health Qigong Association.

5. Center for Taiji \& Qigong Studies.

6. Chen X, Cui J, Li R, Norton R, Park J, et al. (2019) Dao Yin (a.k.a. Qigong): Origin, Development, Potential Mechanisms, and Clinical Applications. Evidence-based complementary and alternative medicine : eCAM, 2019, 3705120 .

7. Chen X, Cui J, Li R, Norton R, Park J, et al. (2019) Dao Yin (a.k.a. Qigong): Origin, Development, Potential Mechanisms, and Clinical Applications. Evidence-based complementary and alternative medicine eCAM: 3705120 .

8. The Chi Center.

9. Ba Duan Jin (2008) Chinese Health Qigong Association. In: Ba Duan Jin (Edt.)., Beijing: Foreign Languages Press, China.

10. Liu Zi Jue (2007) Chinese Health Qigong Association. In: Liu Zi Jue (Edt.)., Beijing: Foreign Languages Press, China.

11. Wu Qin Xi (2008) Chinese Health Qigong Association. In: Wu Qin Xi (Edt.)., Beijing: Foreign Languages Press, China.

12. Yi Jin Jing (2009) Chinese Health Qigong Association. In: Yi Jin Jing (Edt.)., Beijing: Foreign Languages Press, China.

13. Da Wu (2014) Chinese Health Qigong Association. In: Da Wu (Edt.)., Beijing: Foreign Languages Press, China.

14. Shi Er Duan Jin (2014) Chinese Health Qigong Association. In: Shi Er Duan Jin (Edt)., Beijing: Foreign Languages Press, China.

15. Daoyin Yangsheng Gong Shi Er Fa (2014) Chinese Health Qigong Association. In: Daoyin Yangsheng Gong Shi Er Fa (Edt.)., Beijing: Foreign Languages Press, China.

16. Mawanhdui Daoyin Shu (2014) Chinese Health Qigong Association. In: Mawanhdui Daoyin Shu (Edt.)., Beijing: Foreign Languages Press, China.

17. Taiji Yangsheng Zhang (2014) Chinese Health Qigong Association. In: Taiji Yangsheng Zhang (Edt.)., Beijing: Foreign Languages Press, China.

18. Douglas Bill (1999) The Complete Idiot's Guide to T'ai Chi \& QiGong In: Douglas Bill (Edt.)., Alpha Books, a division of Macmillan General Reference, New York.

19. Energy Arts.

20. Frantzis Bruce (2010) Dragon and Tiger Medical Qigong, Volume 1. In: Frantzis Bruce (Edt.)., Fairfax, CA: Energy Arts, USA.

21. Frantzis Bruce (2014) Dragon and Tiger Medical Qigong Volume 2: Qi Cultivation Principles and Exercises. In: Frantzis Bruce (Edt.)., North Atlantic Books, USA.

22. The Institute for Rehabilitative Qigong \& Tai Chi.

23. International Health Qigong Federation.

24. International Medical Tai Chi and Qigong Association.

25. International Qi Gong Association.

26. Jahnke Roger (1997) The Healer Within. Using Traditional Chinese Techniques To Release Your Body's Own Medicine, San Francisco: Harper.

27. Jahnke Roger (2002) The Healing Promise of Qi. New York: Contemporary Books, a division of McGraw-Hill.
28. Johnson Jerry Alan (2000) Chinese Medical Qigong Therapy: A Comprehensive Clinical Guide. In: Johnson Jerry Alan (Edt.)., International Institute of Medical Qigong, Pacific Grove, CA.

29. Johnson Jerry Alan (2005) Chinese Medical Qigong Therapy, Vol. 1: Energetic Anatomy and Physiology. In: Johnson Jerry Alan (Edt.)., International Institute of Medical Qigong, Pacific Grove, CA.

30. Johnson Jerry Alan (2005) Chinese Medical Qigong Therapy, Vol. 2. In: Johnson Jerry Alan (Edt.)., International Institute of Medical Qigong, Pacific Grove, CA.

31. Johnson Jerry Alan (2002) Chinese Medical Qigong Therapy, Vol. 3. In: Johnson Jerry Alan (Edt.)., International Institute of Medical Qigong, Pacific Grove, CA.

32. Johnson Jerry Alan (2002) Chinese Medical Qigong Therapy, Vol. 4: Prescription Exercises and Meditations, Treatment of Internal Diseases, Pediatrics, Geriatrics, Gynecology, Neurology, and Energetic Psychology. In: Johnson Jerry Alan (Edt.)., International Institute of Medical Qigong, Pacific Grove, CA.

33. Johnson Jerry Alan (2005) Chinese Medical Qigong Therapy, Vol. 5: An Energetic Approach to Oncology. In: Johnson Jerry Alan (Edt.)., International Institute of Medical Qigong, Pacific Grove, CA.

34. McGee Robert W (2021) Tai Chi, Qigong and the Treatment of Disease. Biomedical Journal of Scientific \& Technical Research 34(2): 26627 26633.

35. McGee Robert W (2021) Tai Chi, Qigong and Transgender Health. Studies in the Economics of Tai Chi and Qigong 8: 1-24.

36. McGee Robert W (2021) Tai Chi, Qigong and the Treatment of Cancer. Biomedical Journal of Scientific \& Technical Research 34(5): 27173 27182.

37. McGee Robert W (2021) The Use of Yi Jin Jing to Treat Illness: A Summary of Three Studies. Academia Letters, Article 547.

38. McGee Robert W (2021) Tai Chi, Qigong and the Treatment of Depression and Anxiety. Biomedical Journal of Scientific \& Technical Research 36(2): 28350-28354.

39. McGee Robert W (2021) Tai Chi, Qigong and the Treatment of Arthritis. Biomedical Journal of Scientific \& Technical Research 37(5): 2972429734.

40. McGee Robert W (2021) Tai Chi, Qigong and the Treatment of Hypertension. Biomedical Journal of Scientific \& Technical Research 39(1): 31055-31062.

41. McGee Robert W (2020) Qigong: A Bibliography of Books and Other Materials. Studies in the Economics of Qigong 1: 13.

42. McGee Robert W (2020) A Bibliography of Recent Medical Research on Qigong, Fayetteville State University. Studies in the Economics of Qigong $2: 18$.

43. McGee Robert W (2020) Ba Duan Jin as a Treatment for Physical Ailments: A Bibliography of Recent Medical Research. Studies in the Economics of Qigong 3: 11.

44. McGee Robert W (2020) Wu Qin Xi as a Treatment for Physical Ailments: A Bibliography of Recent Medical Research. Studies in the Economics of Qigong 4: 7.

45. McGee Robert W (2020) The Use of Yi Jin Jing to Treat Illness: A Summary of Three Studies. Studies in the Economics of Qigong 5: 8.

46. McGee Robert W (2020) Qigong and the Treatment and Prevention of COVID-19. Studies in the Economics of Qigong 6: 10.

47. McGee Robert W (2020) Qigong and the Treatment and Prevention of Cancer. Studies in the Economics of Qigong 7: 14.

48. PubMed.gov. National Library of Medicine, National Center for Biotechnology Information, National Institute of Health. 
49. Qigong and Energy Medicine Database.

50. The Qigong Research Society.

\section{Spring Forest Qigong.}

52. Tai Chi Healthways.

53. The Qigong Research Society.

54. United States Health Qigong Association.

55. Zhang YP, Hu RX, Han M, Lai BY, Liang SB, et al. (2020) Evidence Base of Clinical Studies on Qi Gong: A Bibliometric Analysis. Complementary therapies in medicine 50: 102392.

56. Xie Y, Guo F, Lu Y, Guo Y, Wei G, et al. (2019) A 12-week Baduanjin Qigong exercise improves symptoms of ankylosing spondylitis: A randomized controlled trial. Complementary therapies in clinical practice 36: 113119.

57. Li Z, Liu S, Wang L, Smith L (2019) Mind-Body Exercise for Anxiety and Depression in COPD Patients: A Systematic Review and Meta-Analysis. International journal of environmental research and public health 17(1): 22.

58. Liu F, Cui J, Liu X, Chen KW, Chen X, et al. (2020) The effect of tai chi and Qigong exercise on depression and anxiety of individuals with substance use disorders: a systematic review and meta-analysis. BMC complementary medicine and therapies 20(1): 161 .

59. Ye J, Simpson MW, Liu Y, Lin W, Zhong W, et al. (2020) The Effects of Baduanjin Qigong on Postural Stability, Proprioception, and Symptoms of Patients With Knee Osteoarthritis: A Randomized Controlled Trial. Frontiers in medicine 6: 307.

60. Ye J, Zheng Q, Zou L, Yu Q, Veronese N, et al. (2020) Mindful Exercise (Baduanjin) as an Adjuvant Treatment for Older Adults (60 Years Old and Over) of Knee Osteoarthritis: A Randomized Controlled Trial. Evidencebased complementary and alternative medicine eCAM: 9869161.

61. Zeng ZP, Liu YB, Fang J, Liu Y, Luo J, et al. (2020) Effects of Baduanjin exercise for knee osteoarthritis: A systematic review and meta-analysis. Complementary therapies in medicine 48: 102279.

62. Duarte L, Gonçalves M, Mendes P, Matos LC, Greten HJ, et al. (2020) Can Qigong improve attention in adolescents? A prospective randomised controlled trial. Journal of bodywork and movement therapies 24(1): 175-181.

63. Rodrigues JM, Mestre M, Fredes LI (2019) Qigong in the treatment of children with autism spectrum disorder: A systematic review. J Integr Med 17(4): 250-260.

64. Zou L, Zhang Y, Yang L, Loprinzi PD, Yeung AS, et al. (2019) Are Mindful Exercises Safe and Beneficial for Treating Chronic Lower Back Pain? A Systematic Review and Meta-Analysis of Randomized Controlled Trials. Journal of clinical medicine 8(5): 628.

65. Liu D, Yi L, Sheng M, Wang G, Zou Y (2020) The Efficacy of Tai Chi and Qigong Exercises on Blood Pressure and Blood Levels of Nitric Oxide and Endothelin-1 in Patients with Essential Hypertension: A Systematic Review and Meta-Analysis of Randomized Controlled Trials. Evidencebased complementary and alternative medicine eCAM: 3267971.

66. Park JE, Yang CS, Jung SY, Kim AR, Lee M, et al. (2019) Exploring the effects of expert-led qigong and self-practice qigong on blood pressure. Integrative medicine research 8(4): 271-272.

67. Arring NM, Barton DL, Brooks T, Zick SM (2019) Integrative Therapies for Cancer-Related Fatigue. Cancer journal (Sudbury, Mass.) 25(5): 349356.

68. Campbell KL, Zadravec K, Bland KA, Chesley E, Wolf F, et al. (2020) The Effect of Exercise on Cancer-Related Cognitive Impairment and
Applications for Physical Therapy: Systematic Review of Randomized Controlled Trials. Physical therapy 100(3): 523-542.

69. Cheng TC, Lee YH, Mar CL, Huang WT, Chang YP (2020) The Health Promoting Mindfulness or Qigong Educational Programs for Beneficial Lifestyle Changes of Cancer Survivors. Journal of cancer education : the official journal of the American Association for Cancer Education 35(4): 743-750.

70. Deng G (2019) Integrative Medicine Therapies for Pain Management in Cancer Patients. Cancer journal (Sudbury, Mass.) 25(5): 343-348.

71. Ford CG, Vowles KE, Smith BW, Kinney AY (2020) Mindfulness and Meditative Movement Interventions for Men Living With Cancer: A Metaanalysis. Annals of behavioral medicine : a publication of the Society of Behavioral Medicine 54(5): 360-373.

72. Garland SN, Mahon K, Irwin MR (2019) Integrative Approaches for Sleep Health in Cancer Survivors. Cancer journal (Sudbury, Mass.) 25(5): 337342.

73. Henshall CL, Allin L, Aveyard H (2019) A Systematic Review and Narrative Synthesis to Explore the Effectiveness of Exercise-Based Interventions in Improving Fatigue, Dyspnea, and Depression in Lung Cancer Survivors. Cancer nursing 42(4): 295-306.

74. Kinney AY, Blair CK, Guest DD, Ani JK, Harding EM, et al. (2019) Biobehavioral effects of Tai Chi Qigong in men with prostate cancer: Study design of a three-arm randomized clinical trial. Contemporary clinical trials communications 16: 100431.

75. Kreutz C, Schmidt ME, Steindorf K (2019) Effects of physical and mind-body exercise on sleep problems during and after breast cancer treatment: a systematic review and meta-analysis. Breast cancer research and treatment 176(1): 1-15.

76. Larkey L, Kim W, James D, Kishida M, Vizcaino M, et al. (2020) MindBody and Psychosocial Interventions May Similarly Affect Heart Rate Variability Patterns in Cancer Recovery: Implications for a Mechanism of Symptom Improvement. Integrative cancer therapies 19: 1534735420949677.

77. Li F, Wang C (2020) "A Good Guy" Again: Biosociality in a Cancer Selfhelp Organization. Medical anthropology, p. 1-14.

78. Lin WF, Zhong MF, Zhou QH, Zhang YR, Wang H, et al. (2019) Efficacy of complementary and integrative medicine on health-related quality of life in cancer patients: a systematic review and meta-analysis. Cancer management and research 11: 6663-6680.

79. Maindet C, Burnod A, Minello C, George B, Allano G, et al. (2019) Strategies of complementary and integrative therapies in cancer-related pain-attaining exhaustive cancer pain management. Supportive care in cancer : official journal of the Multinational Association of Supportive Care in Cancer 27(8): 3119-3132.

80. Oberoi D, Piedalue KL, Pirbhai H, Guirguis S, Santa Mina D, et al. (2020) Factors related to dropout in integrative oncology clinical trials: interim analysis of an ongoing comparative effectiveness trial of mindfulnessbased cancer recovery and Tai chi/Qigong for cancer health (The MATCH study). BMC research notes 13(1): 342 .

81. Osypiuk K, Ligibel J, Giobbie Hurder A, Vergara Diaz G, Bonato P, et al. (2020) Qigong Mind-Body Exercise as a Biopsychosocial Therapy for Persistent Post-Surgical Pain in Breast Cancer: A Pilot Study. Integrative cancer therapies 19: 1534735419893766.

82. Sowada KM (2019) Qigong: Benefits for Survivors Coping With CancerRelated Fatigue. Clinical journal of oncology nursing 23(5): 465-469.

83. Zhang Q Gao X, Liu S, Yu L, Zhu J, et al. (2020) Therapies for cognitive impairment in breast cancer survivors treated with chemotherapy: A protocol for systematic review. Medicine 99(19): e20092. 
84. Zuniga KB, Chan JM, Ryan CJ, Kenfield SA (2020) Diet and lifestyle considerations for patients with prostate cancer. Urologic oncology 38(3): 105-117.

85. Arring NM, Barton DL, Brooks T, Zick SM (2019) Integrative Therapies for Cancer-Related Fatigue. Cancer journal (Sudbury, Mass.) 25(5): 349356.

86. Campbell KL, Zadravec K, Bland KA, Chesley E, Wolf F, et al. (2020) The Effect of Exercise on Cancer-Related Cognitive Impairment and Applications for Physical Therapy: Systematic Review of Randomized Controlled Trials. Physical therapy 100(3): 523-542.

87. Chan J, Deng K, Wu J, Yan JH (2019) Effects of Meditation and Mind-Body Exercises on Older Adults' Cognitive Performance: A Meta-analysis. The Gerontologist 59(6): e782-e790.

88. Ibañez GE, Fennie K, Larkey L (2019) Qigong as a promising mindbody exercise for cognitive functioning: Letter to Editor. Journal of the American Geriatrics Society 67(7): 1533-1534.

89. Garland SN, Mahon K, Irwin MR (2019) Integrative Approaches for Sleep Health in Cancer Survivors. Cancer journal (Sudbury, Mass.) 25(5): 337342.

90. Guo C, Xiang G, Xie L, Liu Z, Zhang X, et al. (2020) Effects of Tai Chi training on the physical and mental health status in patients with chronic obstructive pulmonary disease: a systematic review and metaanalysis. Journal of thoracic disease 12(3): 504-521.

91. Henshall CL, Allin L, Aveyard H (2019) A Systematic Review and Narrative Synthesis to Explore the Effectiveness of Exercise-Based Interventions in Improving Fatigue, Dyspnea, and Depression in Lung Cancer Survivors. Cancer nursing 42(4): 295-306.

92. Larkey L, Kim W, James D, Kishida M, Vizcaino M, et al. (2020) MindBody and Psychosocial Interventions May Similarly Affect Heart Rate Variability Patterns in Cancer Recovery: Implications for a Mechanism of Symptom Improvement. Integrative cancer therapies 19: 1534735420949677.

93. Li Z, Liu S, Wang L, Smith L (2019) Mind-Body Exercise for Anxiety and Depression in COPD Patients: A Systematic Review and Meta-Analysis International journal of environmental research and public health $17(1): 22$.

94. Sowada KM (2019) Qigong: Benefits for Survivors Coping With CancerRelated Fatigue. Clinical journal of oncology nursing 23(5): 465-469.

95. Tong H, Liu Y, Zhu Y, Zhang B, Hu J (2019) The therapeutic effects of qigong in patients with chronic obstructive pulmonary disease in the stable stage: a meta-analysis. BMC complementary and alternative medicine 19(1): 239.

96. Wu C, Tang C (2019) Reply to: Qigong as a Promising Mind-Body Exercise for Cognitive Functioning. Journal of the American Geriatrics Society 67(7): 1534-1535

97. Xie F, Guan C, Cheng Z, Yao F, You Y (2020) Effects of the prolong life with nine turn method (Yan Nian Jiu Zhuan) Qigong on patients with chronic fatigue syndrome: study protocol for a randomized controlled trial. Annals of palliative medicine 9(5): 3571-3583.

98. Yu F, Xin M, Liu N, Huang N, Lu J (2019) The Qigong Wuqinxi for chronic obstructive pulmonary disease: Protocol for a systematic review and meta-analysis. Medicine 98(30): e16633.

99. Zhang Q, Gao X, Liu S, Yu L, Zhu J, et al. (2020) Therapies for cognitive impairment in breast cancer survivors treated with chemotherapy: A protocol for systematic review. Medicine 99(19): e20092.

100. Zhang S, Zhu Q Zhan C, Cheng W, Mingfang X, et al. (2020) Acupressure therapy and Liu Zi Jue Qigong for pulmonary function and quality of life in patients with severe novel coronavirus pneumonia (COVID-19): a study protocol for a randomized controlled trial. Trials 21(1): 751.
101. Zou L, Loprinzi PD, Yeung AS, Zeng N, Huang T (2019) The Beneficial Effects of Mind-Body Exercises for People With Mild Cognitive Impairment: a Systematic Review With Meta-analysis. Archives of physical medicine and rehabilitation 100(8): 1556-1573.

102. Chen JM, Wang ZY, Chen YJ, Ni J (2020) The Application of Eight-Segment Pulmonary Rehabilitation Exercise in People with Coronavirus Disease 2019. Frontiers in physiology 11: 646.

103. Feng F, Tuchman S, Denninger JW, Fricchione GL, Yeung A (2020) Qigong for the Prevention, Treatment, and Rehabilitation of COVID-19 Infection in Older Adults. The American journal of geriatric psychiatry : official journal of the American Association for Geriatric Psychiatry 28(8): 812-819.

104. Zhang S, Zhu Q, Zhan C, Cheng W, Mingfang X, et al. (2020) Acupressure therapy and Liu Zi Jue Qigong for pulmonary function and quality of life in patients with severe novel coronavirus pneumonia (COVID-19): a study protocol for a randomized controlled trial. Trials 21(1): 751.

105. Gill BK, Cant R, Lam L, Cooper S, Lou V (2020) Non-pharmacological depression therapies for older Chinese adults: A systematic review \& meta-analysis. Archives of gerontology and geriatrics 88: 104037.

106. Gouw V, Jiang Y, Seah B, He H, Hong J, et al. (2019) Effectiveness of internal Qigong on quality of life, depressive symptoms and selfefficacy among community-dwelling older adults with chronic disease: A systematic review and meta-analysis. International journal of nursing studies 99: 103378.

107. Henshall CL, Allin L, Aveyard H (2019) A Systematic Review and Narrative Synthesis to Explore the Effectiveness of Exercise-Based Interventions in Improving Fatigue, Dyspnea, and Depression in Lung Cancer Survivors. Cancer nursing 42(4): 295-306.

108. Jin X, Wang L, Liu S, Zhu L, Loprinzi PD, et al. (2019) The Impact of Mind-body Exercises on Motor Function, Depressive Symptoms, and Quality of Life in Parkinson's Disease: A Systematic Review and Metaanalysis. International journal of environmental research and public health 17(1): 31.

109. Lee P, Cai S, Lu EY, Ng B, Jensen MP, et al. (2020) Qigong Reduces Depressive Symptoms of Taiwanese Elderly with Chronic Physical Illness: A Randomized Controlled Trial. Journal of alternative and complementary medicine (New York, N.Y.) 26(1): 76-78.

110. Li Z, Liu S, Wang L, Smith L (2019) Mind-Body Exercise for Anxiety and Depression in COPD Patients: A Systematic Review and Meta-Analysis. International journal of environmental research and public health 17(1): 22.

111. Liu F, Cui J, Liu X, Chen KW, Chen X, et al. (2020) The effect of tai chi and Qigong exercise on depression and anxiety of individuals with substance use disorders: a systematic review and meta-analysis. BMC complementary medicine and therapies 20(1): 161.

112. Lu EY, Lee P, Cai S, So W, Ng B, et al. (2020) Qigong for the treatment of depressive symptoms: Preliminary evidence of neurobiological mechanisms. International journal of geriatric psychiatry 35(11): 1393-1401.

113. So WWY, Cai S, Yau SY, Tsang HWH (2019) The Neurophysiological and Psychological Mechanisms of Qigong as a Treatment for Depression: A Systematic Review and Meta-Analysis. Front Psychiatry 10: 820.

114. Zaprutko T, Göder R, Rybakowski F, Kus K, Kopciuch D, etal. (2020) Nonpharmacological treatments of inpatients with major depression - The case of Polish (Poznan) and German (Kiel) hospital. Complementary therapies in clinical practice 39: 101129 .

115. Sarmento C, Moon S, Pfeifer T, Smirnova IV, Colgrove Y, et al. (2020) The therapeutic efficacy of Qigong exercise on the main symptoms of fibromyalgia: A pilot randomized clinical trial. Integrative medicine research 9(4): 100416. 
116. Liu X, Seah J, Pang B, Tsao MA, Gu F, et al. (2020) A single-arm feasibility study of community-delivered Baduanjin (Qigong practice of the eight Brocades) training for frail older adults. Pilot and feasibility studies 6: 105.

117. Ye J, Simpson MW, Liu Y, Lin W, Zhong W, et al. (2020) The Effects of Baduanjin Qigong on Postural Stability, Proprioception, and Symptoms of Patients With Knee Osteoarthritis: A Randomized Controlled Trial. Frontiers in medicine 6: 307.

118. Ye J, Zheng Q, Zou L, Yu Q, Veronese N, et al. (2020) Mindful Exercise (Baduanjin) as an Adjuvant Treatment for Older Adults (60 Years Old and Over) of Knee Osteoarthritis: A Randomized Controlled Trial. Evidence-based complementary and alternative medicine eCAM: 9869161.

119. Mao S, Zhang X, Chen M, Wang C, Chen Q et al. (2020) Beneficial Effects of Baduanjin Exercise on Left Ventricular Remodelling in Patients after Acute Myocardial Infarction: an Exploratory Clinical Trial and Proteomic Analysis. Cardiovascular drugs and therapy 35(1): 21-32.

120. Oh B, Bae K, Lamoury G, Eade T, Boyle F, et al. (2020) The Effects of Tai Chi and Qigong on Immune Responses: A Systematic Review and MetaAnalysis. Medicines (Basel, Switzerland) 7(7): 39.

121. Chen S, Zhang Y, Wang YT, Liu X, Song W, et al. (2020) The effect of Qigong-based therapy on patients with Parkinson's disease: a systematic review and meta-analysis. Clinical rehabilitation 34(12): 1436-1448.

122. Fidan O, Seyyar GK, Aras B, Colak E, Aras O (2019) The effect of Tai Chi and Qigong on health-related quality of life in Parkinson's disease: a systematic review and meta-analysis of systematic reviews. International journal of rehabilitation research. Internationale Zeitschrift fur Rehabilitationsforschung. Revue internationale de recherches de readaptation 42(3): 196-204

123. Jin X, Wang L, Liu S, Zhu L, Loprinzi PD, et al. (2019) The Impact of Mind-body Exercises on Motor Function, Depressive Symptoms, and Quality of Life in Parkinson's Disease: A Systematic Review and Metaanalysis. International journal of environmental research and public health 17(1): 31 .

124. Li Z, Zhuang J, Jiang Y, Xiao G, Jie K, et al. (2019) Study protocol for a single-blind randomised controlled trial to evaluate the clinical effects of an Integrated Qigong exercise intervention on freezing of gait in Parkinson's disease. BMJ open 9(9): e028869.

125. Moon S, Sarmento C, Steinbacher M, Smirnova IV, Colgrove Y, et al. (2020) Can Qigong improve non-motor symptoms in people with Parkinson's disease - A pilot randomized controlled trial? Complementary therapies in clinical practice 39: 101169.

126. Tang L, Fang Y, Yin J (2019) The effects of exercise interventions on Parkinson's disease: A Bayesian network meta-analysis. Journal of clinical neuroscience: official journal of the Neurosurgical Society of Australasia 70: 47-54.

127. Fidan O, Seyyar GK, Aras B, Colak E, Aras O (2019) The effect of Tai Chi and Qigong on health-related quality of life in Parkinson's disease: a systematic review and meta-analysis of systematic reviews. International journal of rehabilitation research. Internationale Zeitschrift fur Rehabilitationsforschung. Revue internationale de recherches de readaptation 42(3): 196-204.

128. Gouw V, Jiang Y, Seah B, He H, Hong J, et al. (2019) Effectiveness of internal Qigong on quality of life, depressive symptoms and selfefficacy among community-dwelling older adults with chronic disease: A systematic review and meta-analysis. International journal of nursing studies 99: 103378.
129. Jin X, Wang L, Liu S, Zhu L, Loprinzi PD, et al. (2019) The Impact of Mind-body Exercises on Motor Function, Depressive Symptoms, and Quality of Life in Parkinson's Disease: A Systematic Review and Metaanalysis. International journal of environmental research and public health 17(1): 31 .

130. Lin WF, Zhong MF, Zhou QH, Zhang YR, Wang H, et al. (2019) Efficacy of complementary and integrative medicine on health-related quality of life in cancer patients: a systematic review and meta-analysis. Cancer management and research 11: 6663-6680.

131. Sabe M, Sentissi O, Kaiser S (2019) Meditation-based mind-body therapies for negative symptoms of schizophrenia: Systematic review of randomized controlled trials and meta-analysis. Schizophrenia research 212: 15-25.

132. Marks R (2019) Qigong and Musculoskeletal Pain. Current rheumatology reports 21(11): 59.

133. Sabe M, Sentissi O, Kaiser S (2019) Meditation-based mind-body therapies for negative symptoms of schizophrenia: Systematic review of randomized controlled trials and meta-analysis. Schizophrenia research 212: 15-25.

134. Bischoff LL, Otto AK, Hold C, Wollesen B (2019) The effect of physical activity interventions on occupational stress for health personnel: A systematic review. International journal of nursing studies 97: 94-104.

135. Chen CH, Hung KS, Chung YC, Yeh ML (2019) Mind-body interactive qigong improves physical and mental aspects of quality of life in inpatients with stroke: A randomized control study. European journal of cardiovascular nursing : journal of the Working Group on Cardiovascular Nursing of the European Society of Cardiology 18(8): 658-666.

136. Dong X, Zhang R, Guo Y, Chen L, Liu Y, et al. (2020) The efficacy of Qigong exercises for post-stroke mental disorders and sleep disorders: Protocol for a systematic review and meta-analysis. Medicine 99(34): e21784.

137. Wang C, Yu L, Yang J, Wang RW, Zheng YN, et al. (2020) Effectiveness of Liu Zi Jue Qigong versus traditional core stability training for poststroke patients complicated with abnormal trunk postural control: study protocol for a single-center randomized controlled trial. Trials 21(1): 254.

138. Zheng Y, Zhang Y, Li H, Qiao L, Fu W, et al. (2020) Comparative effect of Liuzijue Qigong and conventional respiratory training on trunk control ability and respiratory muscle function in patients at an early recovery stage from stroke: a randomized controlled trial. Archives of physical medicine and rehabilitation 102(3): 423-430.

139. Liu F, Cui J, Liu X, Chen KW, Chen X, et al. (2020) The effect of tai chi and Qigong exercise on depression and anxiety of individuals with substance use disorders: a systematic review and meta-analysis. BMC complementary medicine and therapies 20(1): 161.

140. Tang J, Huang W, Chen X, Lin Q, Wang T, et al. (2019) Liuzijue Qigong: A Voice Training Method For Unilateral Vocal Fold Paralysis Patients. The Annals of otology, rhinology, and laryngology 128(7): 654-661.

141. Wang X, Wu J, Ye M, Wang L, Zheng G (2021) Effect of Baduanjin exercise on the cognitive function of middle-aged and older adults: A systematic review and meta-analysis. Complement Ther Med 59: 102727.

142. Yu L, Liu F, Nie P, Shen C, Chen J, et al. (2021) Systematic review and meta-analysis of randomized controlled trials assessing the impact of Baduanjin exercise on cognition and memory in patients with mild cognitive impairment. Clin Rehabil 35(4): 492-505. 
143. Zheng G, Zheng Y, Xiong Z, Ye B (2020) Effect of Baduanjin exercise on cognitive function in patients with post-stroke cognitive impairment: a randomized controlled trial. Clin Rehabil 34(8): 1028-1039.

144. Li C, Zheng D, Luo J (2021) Effects of traditional Chinese exercise on patients with cognitive impairment: A systematic review and Bayesian network meta-analysis. Nurs Open 8(5): 2208-2220.

145. Chang JY, Tsai PF, Beck C, Hagen JL, Huff DC, et al. (2011) The effect of tai chi on cognition in elders with cognitive impairment. Medsurg Nursing 20(2): 63-70.

146. Tao J, Chen X, Egorova N, Liu J, Xue X, et al. (2017) Tai Chi Chuan and Baduanjin practice modulates functional connectivity of the cognitive control network in older adults. Scientific Reports 7: 41581.

147. Wayne PM, Walsh JN, Taylor Piliae RE, Wells RE, Papp KV, et al. (2014) Effect of Tai Chi on cognitive performance in older adults: Systematic review and meta-analysis. Journal of the American Geriatrics Society 62(1): 25-39.

ISSN: 2574-1241

DOI: 10.26717/BJSTR.2021.40.006424

Robert W McGee. Biomed J Sci \& Tech Res

(C) (P) This work is licensed under Creative

Submission Link: https://biomedres.us/submit-manuscript.php
148. Chen G, Lin Y, Zhao X, Pu B (2021) Effects of Baduanjin on postoperative rehabilitation of patients with breast cancer: A protocol for systematic review and meta-analysis. Medicine (Baltimore) 100(17): e25670.

149. Dai W, Wang X, Xie R, Zhuang M, Chang X, et al. (2020) Baduanjin exercise for cervical spondylotic radiculopathy: A protocol for systematic review and meta-analysis. Medicine (Baltimore) 99(18): e0037s.

150. Li XX, Fan ZJ, Cui J, Lin Q, Zhuang R, et al. (1021) Cardiac rehabilitation of Baduanjin exercise in coronary heart disease after PCI: A protocol for systematic review and meta-analysis of randomized controlled trials. Medicine (Baltimore) 100(15): e25501.

151. Zou L, Zhong C, Xu X, Liu F, Wang C, et al. (2021) Effect of Baduanjin exercise on cervical spondylosis: A protocol for the systematic review of randomized controlled trials. Medicine (Baltimore) 100(12): e24813.

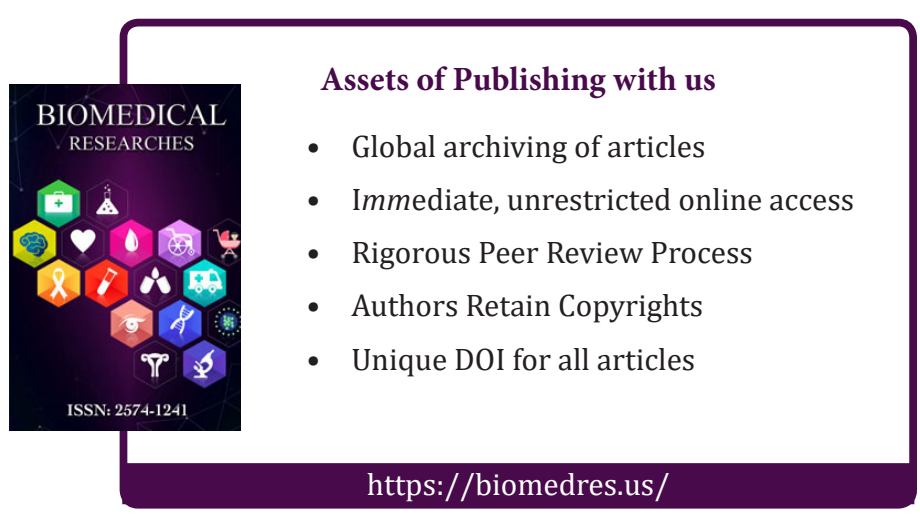

